Research Paper:

\title{
Poor Oral Health-Related Quality of Life in Children With Attention Deficit Hyperactivity Disorder
}

\author{
Arghavan Kamali Sabeti ${ }^{1}$ (D), Sirvan Zarei ${ }^{2}$, Payam Amini ${ }^{3}$ (D) Leila Jahangard $^{4^{*}}$ (D) \\ 1. Department of Pediatric Dentistry, School of Dentistry, Hamadan University of Medical Sciences, Hamadan, Iran. \\ 2. Dentistry Student, School of Dentistry, Hamadan University of Medical Sciences, Hamadan, Iran. \\ 3. Department of Epidemiology, School of Public Health, Hamadan University of Medical Sciences, Hamadan, Iran. \\ 4. Behavioral Disorders and Drug Abuse Research Center, Hamadan University of Medical Sciences, Hamadan, Iran.
}

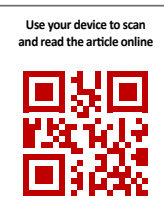

Citation Sabeti A, Zarei S, Amini P, Jahangard L. Poor Oral Health-Related Quality of Life in Children With Attention Deficit Hyperactivity Disorder. Avicenna J of Neuropsychophysiology. 2017; 4(4):131-136. http://dx.doi.org/10.32598/ajnpp.4.4.131

dei http://dx.doi.org/10.32598/ajnpp.4.4.131

(c) (i) (s)

Article info:

Received: 10 Apr 2017

Accepted: 23 Sep 2017

Available Online: 01 Nov 2017

Keywords:

Attention Deficit Hyperactivity Disorder (ADHD), Quality of Life, DMF index

\section{A B STRACT}

Background: Attention Deficit Hyperactivity Disorder (ADHD) is among the most prevalent chronic disorders in children.

Objectives: ADHD is associated with impaired functioning in the social, educational and noneducational areas. This study evaluated the Quality of Life (QoL) related to oral health in ADHD children.

Materials and Methods: In this case-control study, 70 children aged 8-10 years with ADHD disorder were comparatively reviewed with 70 healthy counterparts. The QoL associated with oral health was studied using the Child Perception Questionnaire. The number of decayed (D), missing (M), or filled (F) teeth (T), or Decayed, Missing, and Filled Teeth (DMFT) index were determined in both groups by a dentist (deciduous [dmft] and permanent [DMFT]). The obtained results were analyzed by SPSS.

Results: The mean "dmft" and "DMFT" scores in ADHD children were greater than those of the controls $(\mathrm{P}<0.05)$. The QoL Mean $\pm \mathrm{SD}$ score in ADHD children was greater (worse) than that of the control group ( $49.7 \pm 6.1$ vs. $11.50 \pm 0.88)(P<0.05)$.

Conclusion: Children with ADHD are more vulnerable to dental defects and have lower oral healthrelated QoL; therefore, it is necessary to conduct further interventions in this regard, in addition to the conventional treatment interventions.

\section{* Corresponding Author:}

Leila Jahangard, PhD.

Address: Behavioral Disorders and Drug Abuse Research Center, Hamadan University of Medical Sciences, Hamadan, Iran.

Tel: +98 (913) 3008520

E-mail: jahngard_li_ps@yahoo.com 


\section{Introduction}

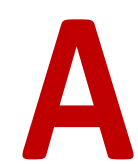

ttention Deficit Hyperactivity Disorder (ADHD) is a prevalent disorder (mean: $5.29 \%$ ) in children worldwide [1]. It can continue until adulthood and often occurs before the age of 7 [2]. Moreover, almost one-third to half of those with ADHD require treatment [3]. The most frequent ADHD symptoms are the inability to concentrate, impulsivity and poor organization [4]. These symptoms can affect the various areas of life, including interpersonal relationships (relationships with siblings, friends, etc.), education, social success, etc.; Even the parents of ADHD children experience difficulties with their children regarding their personal behaviors, such as bathing, dressing, and brushing [5]. Due to various reasons, children at risk have more problems with their teeth [6-9].

Due to problems with attention and concentration in performing daily living activities, like brushing and other oral hygiene routines, they refuse to follow the health guidelines and parental advice [10]. The medications used to treat these children cause dry mouth that following changes in normal salivation, can disrupt oral hygiene and lead to dental damage [11, 12]. These children tend to have increased appetite and consume a large number of sweets, e.g. candy, and confectionaries $[13,14]$. The rate of trauma is higher in this population due to impulsive behaviors [15]. Moreover, considering their behavioral problems, it is more difficult to provide dental care services to them [16-18].

In recent years, more attention has been paid to the Quality of Life (QoL) as a measure for assessing health policies and medical interventions [19]. The concept of QoL refers to the biopsychosocial aspect of health, diseases' effects on wellbeing, and its functional effects on daily life $[18,20]$. A significant aspect of general health assessment is examining peoples' oral health status and its impact on their QoL. Oral Health-Related Quality of Life (OHRQoL) is a multidimensional concept which includes all aspects of health. The OHRQoL reflects the negative impact of oral and dental problems on the QoL. This impact will reduce the QoL through damages to the physical efficiency, social ability, and self-confidence of patients [21].

Hidas et al. argued ADHD patients had a higher plaque and lower saliva flow than healthy controls. However, there was no difference in the Decayed, Missing, and Filled Teeth (DMFT) index between the two groups [12]. Chandra et al. Concluded that dental caries in children with ADHD are higher due to weak oral hygiene and the high consumption of sweets, compared to their healthy counterparts [13]. OHRQoL in children with ADHD has not yet been fully explored and studies have reported different results. Thus, the present study aimed to assess the OHRQoL in children with ADHD.

\section{Materials and Methods}

This was a case-control study. Seventy 8- to 10-yearold children with ADHD referring to Imam Khomeini Medical Clinic (Hamadan City, Iran) from April 2016 to April 2017 included the study participants. The case group members were diagnosed with ADHD by a psychiatrist based on Diagnostic and Statistical Manual of Mental Disorders, $5^{\text {th }}$ Edition (DSM-5) criteria. Moreover, 70 healthy children with matching age and gender were selected as the control group. After obtaining permission from the Education Department of Hamedan, control group members were randomly selected from elementary schools. Considering the health and safety records, they received no diagnosis of ADHD.

The number of Decayed, Missing, and Filled Teeth (DMFT) index were determined in both groups by a dentist (deciduous [dmft] and permanent [DMFT]) using a catheter, mirror, and natural light. To evaluate OHRQoL, the Child Perception Questionnaire $\left(\mathrm{CPQ}^{8-10}\right)$ was used and the questionnaires were completed by the children themselves [22].

$\mathrm{CPQ}$ and clinical oral examination were applied in this study; this included 26 questions in 4 main parts, as follows: oral health (5 items), functional limitations (5 items), emotional health (5 items), and social health (5 items).

Each aspect was recorded in a 5-point Likert-type scale ranging from 0 to 4 ( $0=$ never, $1=$ a few times, $2=$ sometimes, 3=often, 4=everyday). The obtainable OHRQoL scores were 0-140; higher scores indicated the negative effects of oral condition on the QoL which reflects lower QoL. Khadem et al. reported an acceptable validity and reliability for this questionnaire [22].

In this study, taking into account the type 1 error ( $\alpha=0.05$ ) and the study ability equal to $95 \%$, and based on the results of previous studies, the sample size was considered as 70 in each group [23].

The obtained data were analyzed by SPSS. The quantitative variables were presented as Mean \pm SD and the qualitative variables were presented as frequency and 
percentage (\%). To compare the mean QoL scores between the groups, Independent Samples t-test was used. For identifying the correlation between QoL score and DMFT, and dmft, The Pearson's correlation coefficient was used. The significance level was considered at $\mathrm{P}=0.05$.

At the beginning of the study, informed consents were obtained from the children's parents. This study was approved by the Ethics Committee of Hamedan University of Medical Sciences, Hamadan, Iran. Participation or Nonparticipation had no effect on the treatment and care of the patients or the control group.

\section{Results}

The patients with ADHD and the control group did not differ statistically. In total, 33 patients (47.1\%) of the experimental group and $36(54.1 \%)$ of the control group were female. Moreover, $87.7 \%$ of the children's mothers had higher education and $57.7 \%$ had an academic education. The Mean \pm SD age of patients with ADHD and the controls were $8.77 \pm 1.63$ and $9.11 \pm 0.86$ years, respectively. The frequency of employed mothers was significantly higher in the ADHD group. The samples' demographic data are presented in Table 1. The Independent Samples t-test results suggested a statistically significant difference between the two groups, in terms

Table 1. The demographic characteristics of study participants $(n=70)$

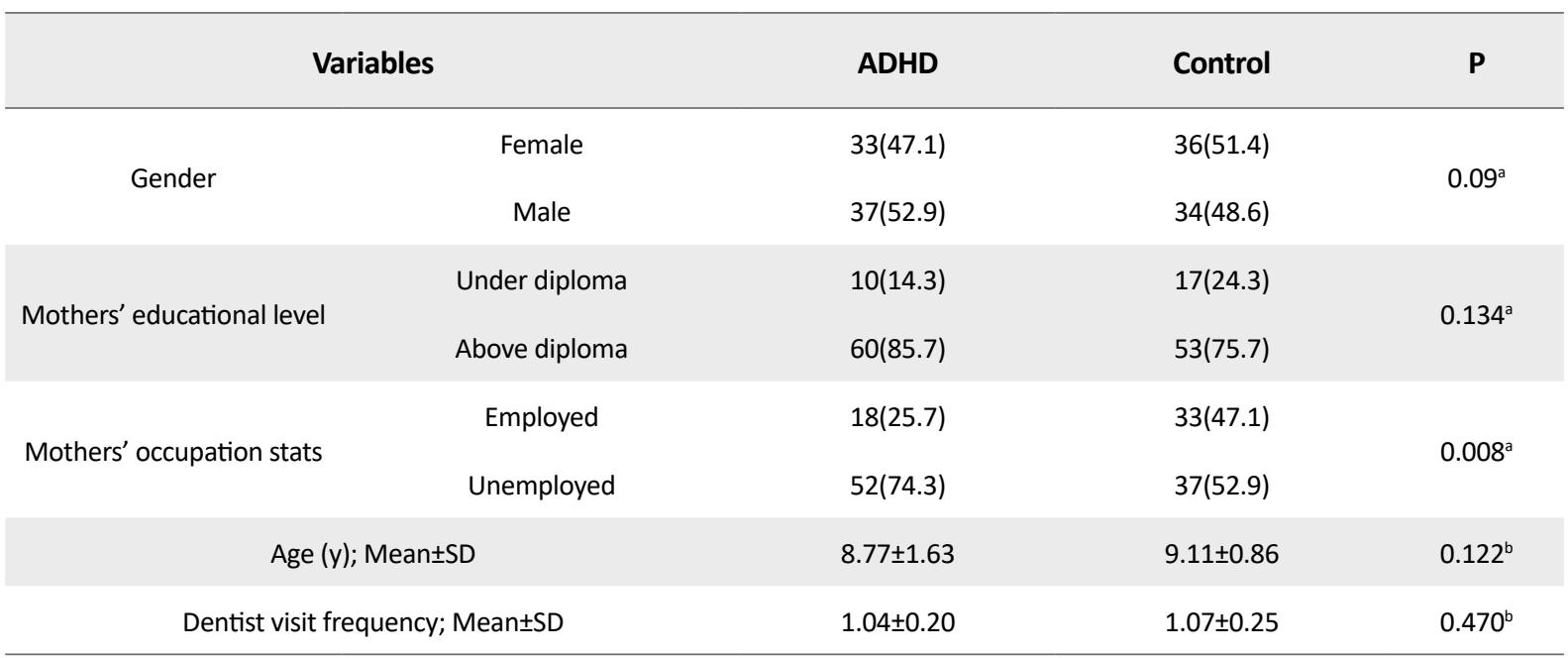

a: Chi-squared test; b: t-test

Table 2. Comparing the $\mathrm{dmft}$ and DMFT scores of study participants $(n=70)$

\begin{tabular}{|c|c|c|c|c|}
\hline & \multirow{2}{*}{ Variables } & \multicolumn{2}{|c|}{ Mean $\pm S D$} & \multirow{2}{*}{$\mathbf{P}$} \\
\hline & & ADHD & Control & \\
\hline & $\mathrm{dmft}(\mathrm{n})$ & $3.4 \pm 0.99$ & $2.4 \pm 0.73$ & 0.001 \\
\hline \multirow{4}{*}{$\mathrm{dmft}$} & Decayed & $2.4 \pm 1.3$ & $1.2 \pm 0.64$ & 0.001 \\
\hline & Missing & $0.61 \pm 0.87$ & $0.73 \pm 0.63$ & 0.001 \\
\hline & Filled & $0.43 \pm 0.92$ & $2.4 \pm 0.73$ & 0.001 \\
\hline & $\mathrm{DMFT}(\mathrm{n})$ & $2.2 \pm 0.86$ & $1.2 \pm 0.66$ & 0.001 \\
\hline \multirow{3}{*}{ DMFT } & Decayed & $2.2 \pm 0.78$ & $1.2 \pm 0.59$ & 0.001 \\
\hline & Missing & $0.11 \pm 0.49$ & - & - \\
\hline & Filled & $2.2 \pm 0.86$ & $1.2 \pm 0.66$ & 0.001 \\
\hline
\end{tabular}


Table 3. The CPQ scores in the case and control groups

\begin{tabular}{|c|c|c|c|c|}
\hline \multirow{2}{*}{ Domain } & \multirow{2}{*}{ Number of Items } & \multicolumn{2}{|c|}{ Mean士SD } & \multirow{2}{*}{$\mathbf{P}$} \\
\hline & & Case & Control & \\
\hline Oral symptoms & 5 & $11.6 \pm 1.4$ & $4.2 \pm 2.5$ & 0.001 \\
\hline Functional limitations & 5 & $10.2 \pm 1.8$ & $2.4 \pm 2.5$ & 0.001 \\
\hline Emotional wellbeing & 5 & $9.8 \pm 1.8$ & $1.9 \pm 1.8$ & 0.001 \\
\hline Social wellbeing & 11 & $18.1 \pm 3.4$ & $2.9 \pm 2.8$ & 0.001 \\
\hline Total CPQ & 26 & $49.7 \pm 6.1$ & $11.5 \pm 0.88$ & 0.001 \\
\hline
\end{tabular}

AJNPP

of mean dmft and DMFT, as well as in all three decayed (D), missing (M), and filled (F) scales (Table 2).

The obtained results revealed a statistically significant difference between the two groups in the mean scores of each of the 4 domains and the total score of OHRQoL. In other words, the mean score of oral health domains, functional limitations, emotional health, and social health in the patients' group was greater than that of the normal group; this data indicates the negative impact of oral problems on QoL (Table 3).

Pearson's correlation coefficient results indicated a positive correlation between the QoL score and the mean dmft ( $r=0.72, P<0.001)$. In fact, with an increase in $\mathrm{dmft}$, the OHRQoL score also enhanced. In other words, with the increase in $\mathrm{dmft}$, the OHRQoL score decreased. Pearson's correlation coefficient outcomes demonstrated a positive correlation between the QoL score and the mean DMFT score $(r=0.52 ; P<0.001)$. Besides, with an increase in DMFT, the OHRQoL score also increased. Accordingly, with the increase in DMFT, the QoL depends on oral health status.

\section{Discussion}

The study findings indicated that the mean $\mathrm{dmft}$ and DMFT scores were significantly higher in the ADHD patients than control subjects; It reflects more dental problems in these patients. Additionally, the patients' QoL was significantly lower than the controls, which indicates the negative impact of dental problems on patients' QoL. An important aspect of general health assessment is examining the oral health status of people and its impact on their QoL [19]. OHRQoL index is an important tool in assessing the health needs and determining the priorities of oral health planning [20].
OHRQoL is also of high importance in children. The prevalence of oral diseases is high in children and the consequent problems negatively impact the quality of their current and future life [20-22]. Tooth decay is the most prevalent chronic disease among children and adolescents around the world. Despite the relative global improvement in oral health, the problems in both developed and developing countries remain challenging. The oral health of ADHD children is affected by several factors, and dental caries and gingivitis are more prevalent in them [8].

They are also more exposed to traumas. There are more issues in the field of behavioral control, and providing dental service to this group is more difficult, especially at lower ages. Therefore, the prevention of oral and dental problems should be the main objective along with the advancement of positive behaviors and acceptance of dental care in this group. The achieved results revealed that the DMFT/dmft index in children with ADHD was significantly greater than that of the healthy group. In addition, there was a statistically significant difference between the two groups in terms of the aforementioned index.

Blomqvist et al., Chandra et al., Grooms et al., and Broadbent et al. also reported a significant statistical difference between DMFT index in children with ADHD and their healthy counterparts [7, 13, 17, 24, 25] However, Hidas et al. and Bimstein et al. studied children with ADHD and reported no significant difference in DMFT index between the healthy and experimental groups $[8,12]$. The lack of a significant difference in the Bimstein study was explained by the small sample size and insufficient evidence.

In the Hidas study, preventive measures in children were the reason for the lack of a significant association 
[12]. Indeed, ADHD children often have difficulty performing daily tasks, like oral hygiene routines due to attention deficit, concentration problems, and functional limitations. These children fail to follow the instructions and guidance of their parents and healthcare providers to maintain oral hygiene. Due to a lack of cooperation and high dental excitability, providing dental treatments to this group is more difficult. Therefore, these can be reasons for the increased rate of decay in ADHD children, compared to healthy ones.

The obtained total OHRQoL questionnaire score, as well as the score of each aspect of the questionnaire, were significantly greater in ADHD children, compared to the controls; thus, higher scores indicated more oral problems in QoL. Therefore, the OHRQoL in ADHD children was significantly lower than the controls. No similar study has been conducted on the OHRQoL in children with ADHD.

In fact, oral and dental problems in ADHD children cause symptoms, such as pain and soreness in the oral cavity, and disturb the normal functioning of the area, which affects their nutritional status. It also affects the level of the child's satisfaction from the way, s/he looks and influences a child's self-esteem, creates concerns in the child, and affects their emotional aspect of life. These problems can also negatively impact the social relationships of these children, i.e. the child is less willing to communicate with others and is less likely to be accepted by peers. In conclusion, oral and dental problems negatively impact all aspects of the lives of ADHD children.

We observed a direct correlation between DMFT/dmft index and OHRQoL scores, where with the increase of DMFT/dmft index, the OHRQoL score also increased. In fact, this data highlights the negative effect of dental caries on OHRQoL which was similar to the study by Do LG et al. and in line with the study by Abanto et al. on children with cerebral palsy $[24,26]$. ADHD children usually do not follow their parents' hygiene instructions; therefore, it seems the mothers' educational level and consciousness level has had no effect on the patients' oral and dental care. Moreover, there was no significant relationship in our findings in normal children either. These obtained data were inconsistent with the studies of Da Matta Felisberto Fernandes et al. [2] and Abanto et al. [27], conducted on healthy children. This difference can be due to the fact that most of the control group mothers in this study had lower educational levels.

Among the limitations of this study, the sample size was almost negligible. The obtained data were limited to one clinic; thus, caution must be considered once generalizing those.

\section{Conclusion}

The OHRQoL score in ADHD children was significantly lower than that of the healthy controls; this finding indicates the negative impact of oral problems on the OHRQoL. There was an inverse association between $\mathrm{DMFT} / \mathrm{dmft}$ index and the QoL in ADHD patients. Therefore, more attention should be paid to ADHD children's oral care and hygiene in addition to the conventional treatments for this disorder.

\section{Data availability}

The SPSS file data used to support the findings of this study are available from the corresponding author upon request.

\section{Ethical Considerations}

\section{Compliance with ethical guidelines}

At the beginning of the study, informed consents were obtained from the children's parents. This study was approved by the Ethics Committee of Hamadan University of Medical Science. Participation or Nonparticipation had no effect on the treatment and care of the patients or the control group.

\section{Funding}

This work was supported by the Vice Chancellor for Research and Technology of Hamadan University of Medical Sciences. The present paper was extracted from the PhD. dissertation of the second author, in School of Dentistry, Hamadan University of Medical Sciences (code: 9412187184).

\section{Authors' contributions}

All authors contributed in preparing this article.

\section{Conflict of interest}

The authors declared no conflict of interest. 


\section{References}

[1] Polanczyk GV, Willcutt EG, Salum GA, Kieling C, Rohde LA. ADHD prevalence estimates across three decades: An updated systematic review and meta-regression analysis. International Journal of Epidemiology. 2014; 43(2):434-42. [DOI:10.1093/ije/dyt261] [PMID] [PMCID]

[2] Friedlander AH. Attention-deficit hyperactivity disorder: Setting the record straight. Special Care in Dentistry. 2004; 24(5):249. [DOI:10.1111/j.1754-4505.2004.tb01700.x] [PMID]

[3] Angold A, Erkanli A, Egger HL, Costello EJ. Stimulant treatment for children: A community perspective. Journal of the American Academy of Child and Adolescent Psychiatry. 2000; 39(8):975-84 [DOI:10.1097/00004583-200008000-00009] [PMID]

[4] American Psychiatric Association. Diagnostic and statistical manua of mental disorders. Washington: American Psychiatric Association; 2000. [DOI:10.1176/appi.books.9780890425596]

[5] Spencer TJ, Biederman J, Mick E. Attention-deficit/hyperactivity disorder: Diagnosis, lifespan, comorbidities, and neurobiology. Journal of Pediatric Psychology. 2007; 32(6):631-42. [DOI:10.1016/j. ambp.2006.07.006] [PMID]

[6] Heitmueller D, Thiering E, Hoffmann U, Heinrich J, Manton D, Kuehnisch J, et al. Is there a positive relationship between molar incisor hypomineralisations and the presence of dental caries? International Journal of Paediatric Dentistry. 2013; 23(2):116-24. [DOI:10.1111/j.1365-263X.2012.01233.x] [PMID]

[7] Broadbent JM, Ayers KM, Thomson WM. Is attention-deficit hyperactivity disorder a risk factor for dental caries? Caries Research. 2004; 38(1):29-33. [DOI:10.1159/000073917] [PMID]

[8] Bimstein E, Wilson J, Guelmann M, Primosch R. Oral characteristics of children with attention-deficit hyperactivity disorder. Special Care in Dentistry. 2008; 28(3):107-10. [DOI:10.1111/j.17544505.2008.00021.x] [PMID]

[9] Sabuncuoglu O. Understanding the relationships between breastfeeding, malocclusion, ADHD, sleep-disordered breathing and traumatic dental injuries. Medical Hypotheses. 2013; 80(3):315-20. [DOI:10.1016/j.mehy.2012.12.017] [PMID]

[10] Efron LA, Sherman JA. Attention deficit disorder: Implications for dental practice. Dentistry Today. 2005; 24(2):134-9. [PMID]

[11] Friedlander AH, Yagiela JA, Mahler ME, Rubin R. The pathophysiology, medical management and dental implications of adult attention-deficit/hyperactivity disorder. Journal of the American Dental Association. 2007; 138(4):475-82. [DOI:10.14219/jada.archive.2007.0199] [PMID]

[12] Hidas A, Noy AF, Birman N, Shapira J, Matot I, Steinberg D, et al. Oral health status, salivary flow rate and salivary quality in children, adolescents and young adults with ADHD. Archives of Oral Biology. 2011; 56(10):1137-41. [DOI:10.1016/j.archoralbio.2011.03.018] [PMID]

[13] Chandra P, Anandakrishna L, Ray P. Caries experience and oral hygiene status of children suffering from attention deficit hyperactivity disorder. Journal of Clinical Pediatric Dentistry. 2009; 34(1):25-9. [DOI:10.17796/jcpd.34.1.n170271832662v44] [PMID]

[14] Beauchaine TP, Gatzke-Kopp LM. Instantiating the multiple levels of analysis perspective in a program of study on externalizing behavior. Development and Psychopathology. 2012; 24(3):1003-18. [DOI:10.1017/S0954579412000508] [PMID] [PMCID]
[15] Sabuncuoglu O. Traumatic dental injuries and attention-deficit/ hyperactivity disorder: Is there a link? Dental Traumatology. 2007; 23(3):137-42. [DOI:10.1111/j.1600-9657.2005.00431.x] [PMID]

[16] Blomqvist M, Augustsson M, Bertlin C, Holmberg K, Fernell E, Dahllof $G$, et al. How do children with attention deficit hyperactivity disorder interact in a clinical dental examination? A video analysis. European Journal of Oral Sciences. 2005; 113(3):203-9. [DOI:10.1111/j.1600-0722.2005.00211.x] [PMID]

[17] Blomqvist M, Holmberg K, Fernell E, Ek U, Dahllof G. Oral health, dental anxiety, and behavior management problems in children with attention deficit hyperactivity disorder. European Journal of Oral Sciences. 2006; 114(5):385-90. [DOI:10.1111/j.16000722.2006.00393.x] [PMID]

[18] Pais-Ribeiro JL. Quality of life is a primary end-point in clinical settings. Clinical Nutrition. 2004; 23(1):121-30. [DOI:10.1016/S02615614(03)00109-2]

[19] Chen MS, Hunter P. Oral health and quality of life in New Zealand: A social perspective. Social Science \& Medicine. 1996; 43(8):121322. [DOI:10.1016/0277-9536(95)00407-6]

[20] Broder HL, McGrath C, Cisneros GJ. Questionnaire development: Face validity and item impact testing of the Child Oral Health Impact Profile. Community Dentistry and Oral Epidemiology. 2007; 35(S1):8-19. [DOI:10.1111/j.1600-0528.2007.00401.x] [PMID]

[21] Miller M, Nevado-Montenegro AJ, Hinshaw SP. Childhood executive function continues to predict outcomes in young adult females with and without childhood-diagnosed ADHD. Journal of Abnormal Child Psychology. 2012; 40(5):657-68. [DOI:10.1007/s10802-011 9599-y] [PMID] [PMCID]

[22] Khadem P, Jabarifar SE, Maryam H, Susan SS, Safaie M. [Evaluation of agreement levels between parents and children in reporting oral health-related quality of life in 11-14 year-old children of Isfahan city in 1389 (Persian)]. Journal of Dental Medicine. 2013; 26(3):185-93

[23] Najarzadegan M, Nejati V, Amiri NA, Sharifian MA. [Effect of cognitive rehabilitation on executive function (working memory and attention) in children with attention deficit hyperactivity disorder (Persian)]. Journal of Rehabilitation Medicine. 2015; 4(2):97-108.

[24] Miller M, Hinshaw SP. Does childhood executive function predict adolescent functional outcomes in girls with ADHD? Journal of Abnormal Child Psychology. 2010; 38(3):315-26. [DOI:10.1007/ s10802-009-9369-2] [PMID] [PMCID]

[25] Grooms MT, Keels MA, Roberts MW, Mclver FT. Caries experience associated with attention-deficit/hyperactivity disorder. Journal of Clinical Pediatric Dentistry. 2005; 30(1):3-7. [DOI:10.17796/ jcpd.30.1.d3n7k5147r3ru571]

[26] Do LG, Spencer A. Oral health-related quality of life of children by dental caries and fluorosis experience. Journal of Public Health Dentistry. 2007; 67(3):132-9. [DOI:10.1111/j.1752-7325.2007.00036.x] [PMID]

[27] Abanto J, Carvalho TS, Bonecker M, Ortega AO, Ciamponi AL, Raggio DP. Parental reports of the oral health-related quality of life of children with cerebral palsy. BMC Oral Health. 2012; 12:15. [DOI:10.1186/1472-6831-12-15] [PMID] [PMCID] 